\title{
Attention Deficits in Alcohol and Drug Use
}

\author{
Behzad Saberi* \\ Medical Research, Iran
}

*Corresponding author: Behzad Saberi MD, Medical Research, Iran.

\section{Minireview}

Attention deficits amount in alcohol use, depends on the alcohol concentration levels in the blood. Higher concentration levels $(0.09 \% \mathrm{~g} / \mathrm{ml})$ are related to higher amounts of attention deficits in comparison with moderate $(0.04 \% \mathrm{~g} / \mathrm{ml})$ concentration levels [1-4].

Increasing in the doses of alcohol is related to the decrease in fMRI BOLD signals in some parts of the brain including Inferior frontal gyrus, Dorsolateral prefrontal cortex and Anterior cingulate cortex.

Acute marijuana administration causes disruption in the visual special attention. Tetrahydrocannabinol (THC) consuming in the marijuana users, results in higher-order executive control tasks impairment according to the Cambridge risk task and Wisconsin card sorting task results. Also, this causes increasing in the Anterior cingulate cortex and right Dorsolateral prefrontal cortex glucose metabolism [5-8].

Visual attention will be enhanced by nicotine use in comparison with marijuana or alcohol use. Also, Dorsolateral prefrontal cortex activations will be increased by nicotine consumption.

Individual differences in gender and abstinence, would modulate the impact of nicotine on the Prefrontal cortex. Studies with using auditory 1-back continuous performance task, have demonstrated this fact.

Since alcohol and drug using would cause various alterations in attention abilities, having knowledge about the related mechanisms involved in such alterations is of importance to deal with the relevant patient's group much more better during clinical practice [9-12].

\section{Acknowledgement}

None.

\section{Conflict of Interest}

No conflict of Interest.

\section{References}

1. Goldstein RZ, Volkow ND (2002) Drug addiction and its underlying neurobiological basis: neuroimaging evidence for the involvement of the frontal cortex. Am J Psychiatry 159(10): 1642-1652.

2. Kringelbach ML, Rolls ET (2004) The functional neuroanatomy of the human orbitofrontal cortex: evidence from neuroimaging and neuropsychology. Prog Neurobiol 72(5): 341-372.

3. Biswal BB, Mennes M, Zuo XN, Gohel S, Kelly C, et al. (2010) Toward discovery science of human brain function. Proc Natl Acad Sci USA 107(10): 4734-4739.

4. Goldstein RZ, Craig AD, Bechara A, Garavan H, Childress AR, et al. (2009) The neurocircuitry of impaired insight in drug addiction. Trends Cogn Sci 13(9): 372-380.

5. Raichle ME, (2001) A default mode of brain function. Proc Natl Acad Sci USA 98: 676-682.

6. Cheetham A, Allen NB, Yucel M, Lubman DI (2010) The role of affective dysregulation in drug addiction. Clin Psychol Rev 30(6): 621-634.

7. Wise RA (1996) Neurobiology of addiction. Curr Opin Neurobiol 6(2): 243-251.

8. Ridderinkhof KR, de Vlugt Y, Bramlage A, Spaan M, Elton M, et al. (2002) Alcohol consumption impairs detection of performance errors in mediofrontal cortex. Science 298(5601): 2209-2211.

9. Myrick H, Anton RF, Li X, Henderson S, Drobes D, et al. (2004) Differential brain activity in alcoholics and social drinkers to alcohol cues: relationship to craving. Neuropsychopharmacology 29(2): 393-402.

10. Russell M (1976) In: Drugs and Drug Dependence. Edwards G (ed), Lexington Books, USA, pp. 182-187.

11. Zubieta JK, Heitzeg MM, Xu Y, Koeppe RA, Ni L, et al. (2005) Regional cerebral blood flow responses to smoking in tobacco smokers after overnight abstinence. Am J Psychiatry 162(3): 567-577.

12. Volkow ND, Fowler JS, Wang GJ (2003) The addicted human brain: insights from imaging studies. J Clin Invest 111(10): 1444-1451. 